\title{
Numerical solution of first order linear fuzzy differential equations using Leapfrog method
}

\author{
S. Sekar ${ }^{1}$, K. Prabhavathi ${ }^{2}$ \\ ${ }^{I}$ (Department of Mathematics, Government Arts College (Autonomous), Salem - 636007 , India) \\ 2 (Department of Mathematics, Bannari Amman Institute of Technology, Sathyamangalam - 638 401, India)
}

\begin{abstract}
This paper presents numerical solutions of first order linear fuzzy differential equations using Leapfrog method. The obtained discrete solutions are compared with single-term Haar wavelet series (STHWS) method [1]. Error graphs and error calculation tables are presented to highlight the efficiency of the Leapfrog method. This method can be implemented in the digital computers and take any time of solutions.
\end{abstract}

Keywords: Fuzzy differential equations, Fuzzy initial value problems, Haar wavelet series, Leapfrog method, Single-term Haar wavelet series method.

\section{Introduction}

S. Abbasbandy and T. Allahviranloo [2] addressed knowledge about dynamical systems modelled by differential equations is often incomplete or vague. It concerns, for example, parameter values, functional relationships, or initial conditions. The well-known methods for solving analytically or numerically initial value problems can only be used for finding a selected system behavior, e.g., by fixing the unknown parameters to some plausible values.

The topics of fuzzy differential equations, which attracted a growing interest for some time, in particular, in relation to the fuzzy control, have been rapidly developed recent years. The concept of a fuzzy derivative was first introduced by S. L. Chang, L. A. Zadeh in [3]. It was followed up by D. Dubois, H. Prade in [4], who defined and used the extension principle. Other methods have been discussed by M. L. Puri, D. A. Ralescu in [5] and R. Goetschel, W. Voxman in [6]. Fuzzy differential equations and initial value problems were regularly treated by O. Kaleva in [7] and [8], S. Seikkala in [9]. A numerical method for solving fuzzy differential equations has been introduced by M. Ma, M. Friedman, A. Kandel in [10] via the standard Euler method.

The structure of this paper is organized as follows. In section 2, the proposed leapfrog method is explained in detailed. In section 3, some basic results on fuzzy numbers and definition of a fuzzy derivative, which have been discussed by S. Seikkala in [9], are given. In section 4, we define the problem that is a fuzzy initial value problem. Its numerical solution is of the main interest of this work. Solving numerically the fuzzy differential equation by the Leapfrog method is discussed in section 5 . The proposed algorithm is illustrated by some examples in section 5 and the conclusion is in section 6.

\section{Leapfrog Method}

The most familiar and elementary method for approximating solutions of an initial value problem is Euler's Method. Euler's Method approximates the derivative in the form of $y^{\prime}=f(t, y), y\left(t_{0}\right)=y_{0}, y \in R^{d}$ by a finite difference quotient $y^{\prime}(t) \approx(y(t+h)-y(t)) / h$. We shall usually discretize the independent variable in equal increments:

$$
t_{n+1}=t_{n}+h, n=0,1, \ldots, t_{0} \text {. }
$$

Henceforth we focus on the scalar case, $N=1$. Rearranging the difference quotient gives us the corresponding approximate values of the dependent variable:

$$
y_{n+1}=y_{n}+h f\left(t_{n}, y_{n}\right), n=0,1, \ldots, t_{0}
$$

To obtain the leapfrog method, we discretize $t_{n}$ as in $t_{n+1}=t_{n}+h, n=0,1, \ldots, t_{0}$, but we double the time interval, $h$, and write the midpoint approximation $y(t+h)-y(t) \approx h y^{\prime}\left(t+\frac{h}{2}\right)$ in the form

$$
y^{\prime}(t+h) \approx(y(t+2 h)-y(t)) / h
$$

and then discretize it as follows:

$$
y_{n+1}=y_{n-1}+2 h f\left(t_{n}, y_{n}\right), n=0,1, \ldots, t_{0}
$$


The leapfrog method is a linear $m=2$-step method, with $a_{0}=0, a_{1}=1, b_{-1}=-1, b_{0}=2$ and $b_{1}=0$. It uses slopes evaluated at odd values of $n$ to advance the values at points at even values of $n$, and vice versa, reminiscent of the children's game of the same name. For the same reason, there are multiple solutions of the leapfrog method with the same initial value $y=y_{0}$. This situation suggests a potential instability present in multistep methods, which must be addressed when we analyze them-two values, $y_{0}$ and $y_{1}$, are required to initialize solutions of $y_{n+1}=y_{n-1}+2 h f\left(t_{n}, y_{n}\right), n=0,1, \ldots, t_{0}$ uniquely, but the analytical problem $y^{\prime}=f(t, y), y\left(t_{0}\right)=y_{0}, y \in R^{d}$ only provides one. Also for this reason, one-step methods are used to initialize multistep methods.

\section{Preliminaries}

A parallelogram fuzzy number $u$ is defined by four real numbers $k<l<m<n$, where the base of the parallelogram is the interval $[k, n]$ and its vertices at $x=l, x=m$. Parallelogram fuzzy number will be written as $u=(k, l, m, n)$. The membership function for the parallelogram fuzzy number $u=(k, \square \square m, n)$ is defined as the following:

$$
u(x)= \begin{cases}\frac{x-k}{l-k}, & k \leq x \leq l \\ 1, & l \leq x \leq m \quad \text { u } \\ \frac{x-n}{m-n}, & m \leq x \leq n\end{cases}
$$

we will have: (1) $u>0$ if $k>0$; (2) $u>0$ if $>0$; (3) $u>0$ if $m>0$ \& (4) $u>0$ if $n>0$. Let us denote $\mathrm{R}_{\mathrm{F}}$ by the class of all fuzzy subsets of $\mathrm{R}$ (i.e. $u: \mathrm{R} \rightarrow[0,1]$ ) satisfying the following properties:

(i) $\forall u \in R_{F}, u$ is normal, i.e. $\exists x_{0} \in R$ with $u\left(x_{0}\right)=1$.

(ii) $\forall u \in R_{F}, u$ is convex fuzzy set,

$$
\text { i.e. } u(t x+(1-t) y) \geq \min \{u(x, u(y))\}, \forall t \in[0,1], x, y \in R \text {. }
$$

(iii) $\forall u \in R_{F}, u$ is upper semi continuous on $\mathrm{R}$.

(iv) $\{\overline{x \in R ; u(x)>0}\}$ is compact, where $\bar{A}$ denotes the closure of $A$.

Then $\mathrm{R}_{\mathrm{F}}$ is called the space of fuzzy numbers .Obviously $\mathrm{R} \square \square \mathrm{R}$. Here $\mathrm{R} \square \square \mathrm{R}$ is understood as $\mathrm{R}=$ $\{\square\{x\} ; x$ is usual real number $\}$. We define the r-level set, $x \square \square R$;

$[u] r=\{x \mid u(x) \square \square r\}, 0 \square \square r \square \square l ;$

(2)

Clearly, $[u]_{0}=\{x \mid u(x)>0\}$ is compact, which is a closed bounded interval and we denote by $[u] \mathrm{r}=$ $[u(r), u(r)]$. It is clear that the following statements are true.

1. $\underline{u}(r)$ is a bounded left continuous non decreasing function over [0,1],

2. $\bar{u}(r)$ is a bounded right continuous non increasing function over $[0,1]$,

3. $\underline{u}(r) \square \square \bar{u}(r)$ for all $r \square \square(01$ ], for more details see [2],[3].

Let $D: R_{F} \square \square \mathrm{R} \rightarrow R+\mathrm{U} \square 0 \square$, $D(u, v)=\operatorname{Sup}_{r \square \square[0,19 \max } \square \square|\underline{u}(r) \square \square \underline{v}(r)|,|\bar{u}(r) \square \square \bar{v}(r)| \square$, be Hausdorff distance between fuzzy numbers, where $[u]_{\mathrm{r}}=[\underline{u}(r), \bar{u}(r)],[v]_{\mathrm{r}}=[\underline{v}(r), \bar{v}(r)]$. The following properties are well-known:

$D(u+w, v+w)=D(u, v), \forall u, v, w \in R_{F}$,

$D(k . u, k . v)=|k| D(u, v), \forall k \in R, u, v \in R_{F}$,

$D(u+v, w+e) \leq D(u, w)+D(v, e), \forall u, v, w, e \in R_{F}$ and $\left(R_{F}, \mathrm{D}\right)$ is a complete metric space. 


\section{Fuzzy Initial Value Problems}

Consider a first-order fuzzy initial value differential equation is given by

$$
\left.\begin{array}{l}
y^{\prime}(t)=f(t, y(t)), t \in\left[t_{0}, T\right], \\
y\left(t_{0}\right)=y_{0},
\end{array}\right\}
$$

where $\mathrm{y}$ is a fuzzy function of $\mathrm{t}, \mathrm{f}(\mathrm{t}, \mathrm{y})$ is a fuzzy function of the crisp variable $\mathrm{t}$ and the fuzzy variable $y, y^{\prime}$ is the fuzzy derivative of $\mathrm{y}$ and $y\left(t_{0}\right)=y_{0}$ is a parallelogram or a parallelogram shaped fuzzy number. We denote the fuzzy function y by $y=\lfloor y, \bar{y}]$. It means that the r-level set of $\mathrm{y}(t)$ for $t \in\left[t_{0}, T\right]$ is

$$
[y(t)]_{r}=\lfloor y(t ; r), \bar{y}(t ; r)\rfloor\left[y\left(t_{0}\right)\right]_{r}=\left\lfloor\underline{y}\left(t_{0} ; r\right), \bar{y}\left(t_{0} ; r\right)\right\rfloor r \in(0,1]
$$

we write $f(t ; y)=[\underline{f}(t ; y), \bar{f}(t ; y)]$ and $\underline{f}(t ; y)=F[t, \underline{y}, \bar{y}] \bar{f}(t ; y)=G[t, \underline{y}, \bar{y}]$.

Because of $y^{\prime}(t)=f(t, y)$ we have

$$
\begin{aligned}
& f(t ; y(t ; r))=F[t ; \underline{y}(t ; r), \bar{y}(t ; r)] \\
& \bar{f}(t ; y(t ; r))=G[t ; \underline{y}(t ; r), \bar{y}(t ; r)]
\end{aligned}
$$

By using the extension principle, we have the membership function

$$
f(t ; y(t))(s)=\operatorname{Sup}\{y(t)(\tau) \mid s=f(t, \tau)\}, s \in R
$$

so fuzzy number $f(t ; y(t))$. From this it follows that

$$
\begin{aligned}
& {[f(t ; y(t))]_{r}=[f(t, y(t ; r)), \bar{f}(t, y(t ; r))]_{,} r \in[0 ; 1]} \\
& \text { where } f(t, y(t ; r))=\min \left\{f(t, u) \backslash u \in[y(t)]_{r}\right\} \\
& \bar{f}(t, y(t ; r))=\max \left\{f(t, u) \backslash u \in[y(t)]_{r}\right\}
\end{aligned}
$$

Definition 4.1

A function $\mathrm{f}: \mathrm{R} \rightarrow \mathrm{R}_{\mathrm{F}}$ is said to be fuzzy continuous function, if for an arbitrary fixed $t_{0} \in R$ and $\in>0, \delta>0$ such that $\mid t-t_{0}<\delta \Rightarrow D\left[f(t), f\left(t_{0}\right)\right]<\in$ exists.

Throughout this paper we also consider fuzzy functions which are continuous in metric $D$. Then the continuity of $f(t, y(t) ; r)$ guarantees the existence of the definition of $f(t, y(t) ; r)$ for $t \in\left[t_{0}, T\right]$ and $r \in[0,1]$ [1]. Therefore, the functions $G$ and $F$ can be definite too.

\section{Numerical Examples}

Consider a first-order fuzzy initial value differential equation is given by In this section, the exact solutions and approximated solutions obtained by Leapfrog method and STHWS method. To show the efficiency of the Leapfrog method, we have considered the following problem taken from [1], along with the exact solutions.

The discrete solutions obtained by the two methods, Leapfrog method and STHWS method; the absolute errors between them are tabulated and are presented in Table 1 and Table 2. To distinguish the effect of the errors in accordance with the exact solutions, graphical representations are given for selected values of " $r$ " and are presented in Fig. 1 to Fig. 6 for the following problem, using three dimensional effects.

Example 5.1

Consider the initial value problem [1]

$$
\left.\begin{array}{l}
y^{\prime}(t)=t f(t), t \in[0,1], \\
y(0)=(1.01+0.1 r \sqrt{e}, 1.5+0.1 r \sqrt{e})
\end{array}\right\}
$$

The exact solution at $t=0.1$ is given by

$$
Y(0.1 ; r)=\left\lfloor(1.01+0.1 r \sqrt{e}) e^{0.005},(1.5+0.1 r \sqrt{e}) e^{0.005}\right\rfloor, 0 \leq r \leq 1
$$

Example 5.2

Consider the fuzzy initial value problem [1] 


$$
\left.\begin{array}{l}
y^{\prime}(t)=y(t), t \in I=[0,1], \\
y(0)=(0.75+0.25 r, 1.125-0.125 r), 0<r \leq 1 .
\end{array}\right\}
$$

The exact solution is given by

$$
\begin{aligned}
& Y_{1}(t ; r)=y_{1}(0 ; r) e^{t}, Y_{2}(t ; r)=y_{2}(0 ; r) e^{t} \text { which at } t=1 \\
& Y_{1}(1 ; r)=[(0.75+0.25 r) e,(1.125-0.125 r) e], 0<r \leq 1 .
\end{aligned}
$$

Example 5.3

Consider the fuzzy initial value problem [1]

$$
y^{\prime}(t)=c_{1} y^{2}(t)+c_{2}, y(0)=0
$$

where $c_{i}>0$, for $i=1,2$ are triangular fuzzy numbers.

The exact solution is given by

$$
\begin{aligned}
& Y_{1}(t ; r)=l_{1}(r) \tan \left(w_{1}(r) t\right), \\
& Y_{2}(t ; r)=l_{2}(r) \tan \left(w_{2}(r) t\right),
\end{aligned}
$$

with

$$
\begin{aligned}
& l_{1}(r)=\sqrt{c_{2,1}(r) / c_{1,1}(r)}, \quad l_{2}(r)=\sqrt{c_{2,2}(r) / c_{1,2}(r)} \\
& w_{1}(r)=\sqrt{c_{1,1}(r) / c_{2,1}(r)}, \quad w_{2}(r)=\sqrt{c_{1,2}(r) / c_{2,2}(r)}
\end{aligned}
$$

\begin{tabular}{|c|c|c|c|c|c|c|}
\hline \multirow[b]{3}{*}{$\mathrm{r}$} & \multicolumn{6}{|c|}{ STHWS Error } \\
\hline & \multicolumn{2}{|c|}{ Example 5.1} & \multicolumn{2}{|c|}{ Example 5.2} & \multicolumn{2}{|c|}{ Example 5.3} \\
\hline & $y_{1}$ & $y_{2}$ & $y_{1}$ & $y_{2}$ & $y_{1}$ & $y_{2}$ \\
\hline 0.1 & $1.00 \mathrm{E}-07$ & $1.00 \mathrm{E}-07$ & $6.00 \mathrm{E}-07$ & $6.00 \mathrm{E}-07$ & $1.00 \mathrm{E}-07$ & $1.00 \mathrm{E}-08$ \\
\hline 0.2 & $2.00 \mathrm{E}-07$ & $2.00 \mathrm{E}-07$ & $7.00 \mathrm{E}-07$ & $7.00 \mathrm{E}-07$ & $2.00 \mathrm{E}-07$ & $2.00 \mathrm{E}-08$ \\
\hline 0.3 & $3.00 \mathrm{E}-07$ & $3.00 \mathrm{E}-07$ & $8.00 \mathrm{E}-07$ & $8.00 \mathrm{E}-07$ & $3.00 \mathrm{E}-07$ & $3.00 \mathrm{E}-08$ \\
\hline 0.4 & $4.00 \mathrm{E}-07$ & $4.00 \mathrm{E}-07$ & $9.00 \mathrm{E}-07$ & $9.00 \mathrm{E}-07$ & $4.00 \mathrm{E}-07$ & $4.00 \mathrm{E}-08$ \\
\hline 0.5 & $5.00 \mathrm{E}-07$ & $5.00 \mathrm{E}-07$ & $1.00 \mathrm{E}-06$ & $1.00 \mathrm{E}-06$ & $5.00 \mathrm{E}-07$ & $5.00 \mathrm{E}-08$ \\
\hline 0.6 & $6.00 \mathrm{E}-07$ & $6.00 \mathrm{E}-07$ & $1.10 \mathrm{E}-06$ & $1.10 \mathrm{E}-06$ & $6.00 \mathrm{E}-07$ & $6.00 \mathrm{E}-08$ \\
\hline 0.7 & $7.00 \mathrm{E}-07$ & $7.00 \mathrm{E}-07$ & $1.20 \mathrm{E}-06$ & $1.20 \mathrm{E}-06$ & $7.00 \mathrm{E}-07$ & $7.00 \mathrm{E}-08$ \\
\hline 0.8 & $8.00 \mathrm{E}-07$ & $8.00 \mathrm{E}-07$ & $1.30 \mathrm{E}-06$ & $1.30 \mathrm{E}-06$ & $8.00 \mathrm{E}-07$ & $8.00 \mathrm{E}-08$ \\
\hline 0.9 & $9.00 \mathrm{E}-07$ & $9.00 \mathrm{E}-07$ & $1.40 \mathrm{E}-06$ & $1.40 \mathrm{E}-06$ & $9.00 \mathrm{E}-07$ & $9.00 \mathrm{E}-08$ \\
\hline 1 & $1.00 \mathrm{E}-06$ & $1.00 \mathrm{E}-06$ & $1.50 \mathrm{E}-06$ & $1.50 \mathrm{E}-06$ & $1.00 \mathrm{E}-06$ & $9.90 \mathrm{E}-08$ \\
\hline
\end{tabular}

where

$$
\begin{aligned}
& {\left[c_{1}\right]_{r}=\left\lfloor c_{1,1}(r), c_{1,2}(r)\right\rfloor \text { and }\left[c_{2}\right]_{r}=\left\lfloor c_{2,1}(r), c_{2,2}(r)\right\rfloor} \\
& c_{1,1}(r)=0.5+0.5 r, c_{1,2}(r)=1.5-0.5 r, \\
& c_{2,1}(r)=0.75+0.25 r, c_{2,2}(r)=1.25-0.25 r,
\end{aligned}
$$

The r-level sets of $y^{\prime}(t)$ are

$$
\begin{aligned}
& Y_{1}^{\prime}(t ; r)=c_{2,1}(r) \sec ^{2}\left(w_{1}(r) t\right), \\
& Y_{2}^{\prime}(t ; r)=c_{2,2}(r) \sec ^{2}\left(w_{2}(r) t\right),
\end{aligned}
$$

which defines a fuzzy number. We have

$$
\begin{aligned}
& f_{1}(t, y ; r)=\min \left\{c_{1} \cdot u^{2}+c_{2} \mid u \in\left[y_{1}(t ; r), y_{2}(t ; r)\right], c_{1} \in\left[c_{1,1}(r), c_{1,2}(r)\right], c_{2} \in\left[c_{2,1}(r), c_{2,2}(r)\right]\right\}, \\
& f_{2}(t, y ; r)=\max \left\{c_{1} \cdot u^{2}+c_{2} \mid u \in\left[y_{1}(t ; r), y_{2}(t ; r)\right], c_{1} \in\left[c_{1,1}(r), c_{1,2}(r)\right], c_{2} \in\left[c_{2,1}(r), c_{2,2}(r)\right]\right\} .
\end{aligned}
$$

Table 1: Error Calculations

Table 2: Error Calculations

\begin{tabular}{|c|c|c|c|c|c|c|}
\hline & \multicolumn{7}{|c|}{ Leapfrog Error } \\
\hline \multirow{3}{*}{$\mathrm{r}$} & \multicolumn{2}{|c|}{ Example 5.1 } & \multicolumn{2}{c|}{ Example 5.2 } & \multicolumn{2}{c|}{ Example 5.3 } \\
\cline { 2 - 7 } & $y_{1}$ & $y_{2}$ & $y_{1}$ & $y_{2}$ & $y_{1}$ & $y_{2}$ \\
\hline 0.1 & $1.00 \mathrm{E}-07$ & $1.00 \mathrm{E}-07$ & $6.00 \mathrm{E}-07$ & $6.00 \mathrm{E}-07$ & $1.00 \mathrm{E}-07$ & $1.00 \mathrm{E}-08$ \\
\hline 0.2 & $2.00 \mathrm{E}-07$ & $2.00 \mathrm{E}-07$ & $7.00 \mathrm{E}-07$ & $7.00 \mathrm{E}-07$ & $2.00 \mathrm{E}-07$ & $2.00 \mathrm{E}-08$ \\
\hline 0.3 & $3.00 \mathrm{E}-07$ & $3.00 \mathrm{E}-07$ & $8.00 \mathrm{E}-07$ & $8.00 \mathrm{E}-07$ & $3.00 \mathrm{E}-07$ & $3.00 \mathrm{E}-08$ \\
\hline
\end{tabular}




\begin{tabular}{|l|l|l|l|l|l|l|}
\hline 0.4 & $4.00 \mathrm{E}-07$ & $4.00 \mathrm{E}-07$ & $9.00 \mathrm{E}-07$ & $9.00 \mathrm{E}-07$ & $4.00 \mathrm{E}-07$ & $4.00 \mathrm{E}-08$ \\
\hline 0.5 & $5.00 \mathrm{E}-07$ & $5.00 \mathrm{E}-07$ & $1.00 \mathrm{E}-06$ & $1.00 \mathrm{E}-06$ & $5.00 \mathrm{E}-07$ & $5.00 \mathrm{E}-08$ \\
\hline 0.6 & $6.00 \mathrm{E}-07$ & $6.00 \mathrm{E}-07$ & $1.10 \mathrm{E}-06$ & $1.10 \mathrm{E}-06$ & $6.00 \mathrm{E}-07$ & $6.00 \mathrm{E}-08$ \\
\hline 0.7 & $7.00 \mathrm{E}-07$ & $7.00 \mathrm{E}-07$ & $1.20 \mathrm{E}-06$ & $1.20 \mathrm{E}-06$ & $7.00 \mathrm{E}-07$ & $7.00 \mathrm{E}-08$ \\
\hline 0.8 & $8.00 \mathrm{E}-07$ & $8.00 \mathrm{E}-07$ & $1.30 \mathrm{E}-06$ & $1.30 \mathrm{E}-06$ & $8.00 \mathrm{E}-07$ & $8.00 \mathrm{E}-08$ \\
\hline 0.9 & $9.00 \mathrm{E}-07$ & $9.00 \mathrm{E}-07$ & $1.40 \mathrm{E}-06$ & $1.40 \mathrm{E}-06$ & $9.00 \mathrm{E}-07$ & $9.00 \mathrm{E}-08$ \\
\hline 1 & $1.00 \mathrm{E}-06$ & $1.00 \mathrm{E}-06$ & $1.50 \mathrm{E}-06$ & $1.50 \mathrm{E}-06$ & $1.00 \mathrm{E}-06$ & $9.90 \mathrm{E}-08$ \\
\hline
\end{tabular}

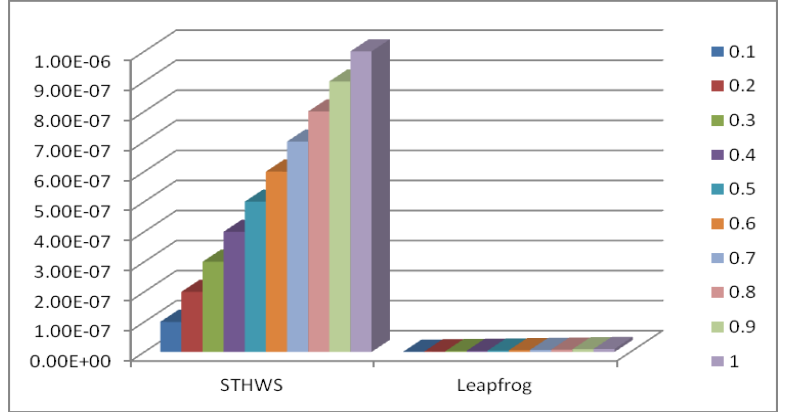

Fig. 1 Error estimation of Example 5.1 at $\mathrm{y}_{1}$

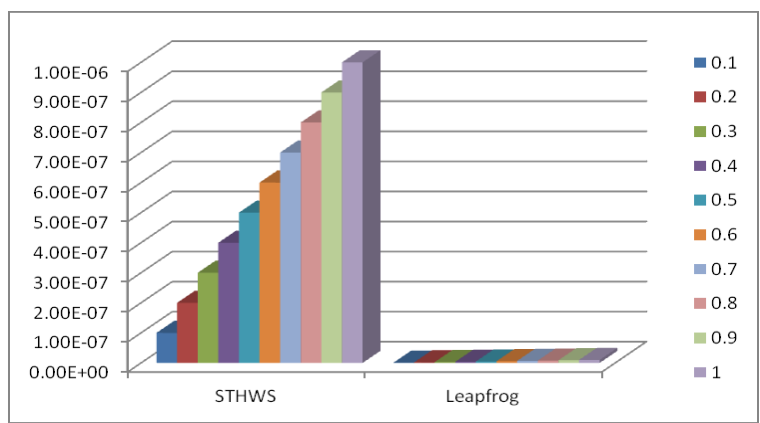

Fig. 2 Error estimation of Example 5.1 at $\mathrm{y}_{2}$

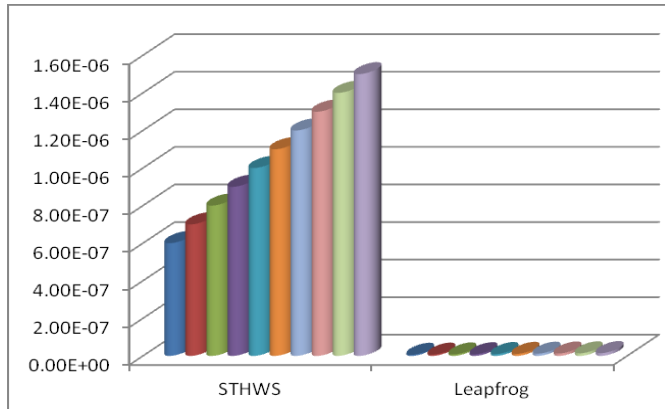

Fig. 3 Error estimation of Example 5.2 at $\mathrm{y}_{1}$

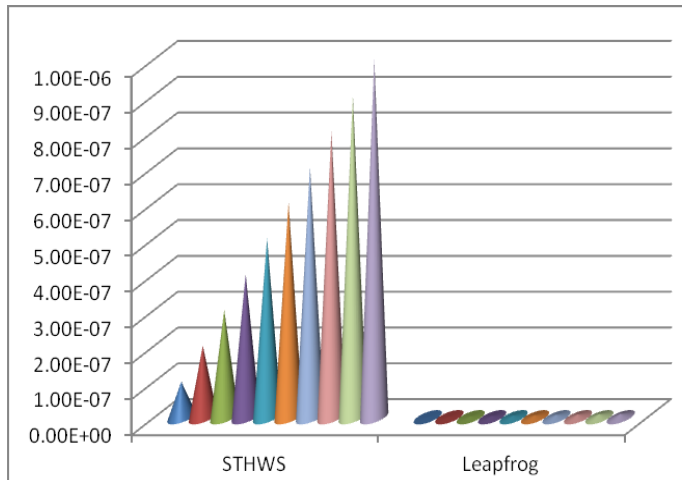

Fig. 5 Error estimation of Example 5.3 at $\mathrm{y}_{1}$

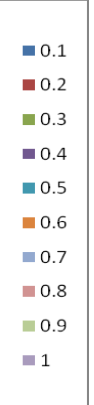

0.3

0.4

0.7

0.9

1

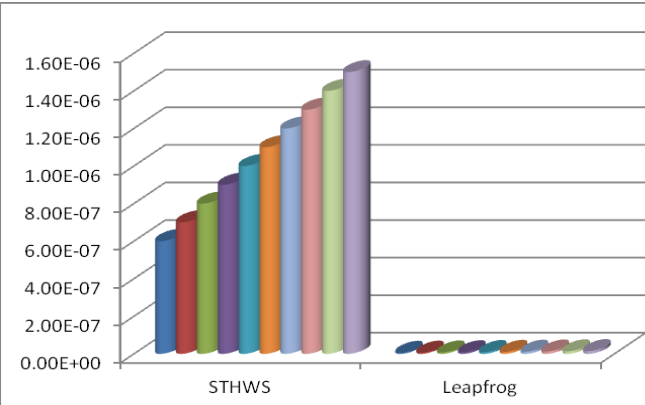

Fig. 4 Error estimation of Example 5.2 at $y_{2}$

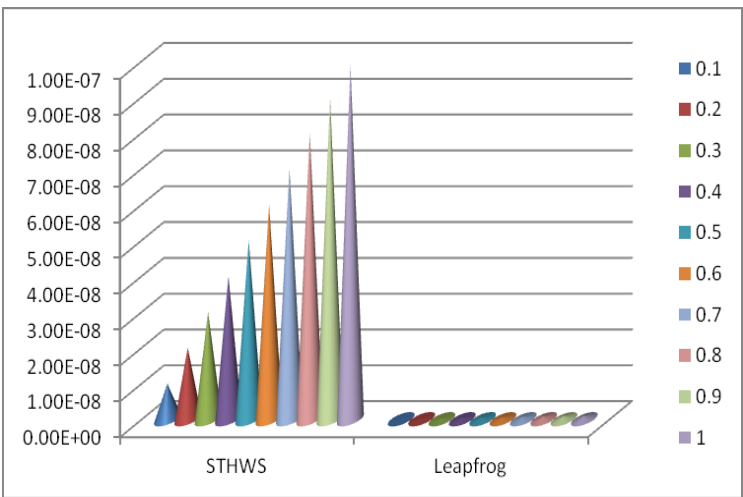

Fig. 6 Error estimation of Example 5.3 at $\mathrm{y}_{2}$

\section{Conclusion}

In this paper, the Leapfrog method has been successfully employed to obtain the approximate analytical solutions of the first order linear fuzzy differential equations. Compare to STHWS method, Leapfrog method gives less error from the Table 1 and Table 2. Also it is clear that from the Fig. 1 to Fig. 6 the Leapfrog method introduced in Section 2 performs better than STHWS method.

\section{Acknowledgements}

The authors gratefully acknowledge the Dr. A. Murugesan, Assistant Professor, Department of Mathematics, Government Arts College (Autonomous), Cherry Road, Salem - 636 007, for encouragement and support. The authors also thank Dr. S. Mehar Banu, Assistant Professor, Department of Mathematics, Government Arts College for Women (Autonomous), Salem - 636 008, Tamil Nadu, India, for her kind help and suggestions. 


\section{References}

[1] S.Sekar and S.Senthilkumar, Single-term Haar wavelet series for fuzzy differential equations, International Journal of Mathematics Trends and Technology, 4(9), 2013, $181-188$

[2] S. Abbasbandy and T. Allahviranloo, Numerical solutions of fuzzy differential equations by Taylor method, Journal of Computational Methods in Applied Mathematics. 2, 2002, 113-124.

[3] S. S. L. Chang and L. A. Zadeh, On fuzzy mapping and control, IEEE Transactions on Systems, Man, and Cybernetics, 2, 1972, 3034.

[4] D. Dubois and H. Prade, Towards fuzzy differential calculus.III. Differentiation, Fuzzy Sets and Systems, 8( 3), 1982, 225-233.

[5] M. L. Puri and D. A. Ralescu, Differentials of fuzzy functions, Journal of Mathematical Analysis and Applications, 91(2), 1983, $552-558$.

[6] R. Goetschel and Woxman, Elementary fuzzy calculus, Fuzzy Sets and Systems, 18, 1986, 31-43.

[7] O. Kaleva, Fuzzy differential equations, Fuzzy Sets and Systems, 24(3), 1987, 301-317.

[8] O. Kaleva, The Cauchy problem for fuzzy differential equations, Fuzzy Sets and Systems, 35(3), $1990,389-396$.

[9] S. Seikkala, On the fuzzy initial value problem, Fuzzy Sets and Systems, 24(3), 1987, 319-330.

[10] M. Ma, M. Friedman and A. kandel, Numerical Solutions of Fuzzy Differential Equations, Fuzzy Sets and Systems, 105, 1999, 133138 . 